\title{
A low-cost solid state device for producing delayed auditory feedback
}

\author{
FRED L. YAFFE \\ Washburn University, Topeka, Kansas 66621 \\ and \\ LAURENCE BOSTWICK \\ Bostwick Electronics, Topeka, Kansas 66603
}

\begin{abstract}
This paper presents a design for construction of a relatively low-cost solid state delayed auditory feedback device. The advantages of this unit are that it is reliable, flexible, easy to operate, and it does not require audio tape.
\end{abstract}

When the regular auditory feedback from selfproduced speech is delayed, the normal individual usually experiences difficulty in speaking smoothly. This phenomenon, known as delayed auditory feedback (DAF), although disruptive for normal speakers, has been used for the treatment of stuttering as well as other speech difficulties (Allen, Antonitis, \& Magaro, 1978; Lozano \& Dreyer, 1978; Martin \& Haroldson, 1979; Stephen \& Haggard, 1980; Timmons \& Boudreau, 1978a, 1978b).

The most pronounced effects of DAF occur when speech is delayed around $250 \mathrm{msec}$, with most studies varying the delay from 3 to $750 \mathrm{msec}$ (Allen et al., 1978; Doehrman, Landau, \& O'Connell, 1978; Lechner, 1979; Martin \& Haroldson, 1979). Effects of various delay times seem to be dependent on age and, probably, fundamental frequencies. The primary instrument action used to produce DAF consists of a stereo tape recorder with two or three heads. As the tape passes the first head, the voice is recorded. It is then played back from the second head and erased by the third head. Various DAF times are acquired by altering the distances between the first and second recorder heads in combination with the various tape drive speeds available (Collins \& Worthington, 1978; Elias, 1977; Lechner, 1979; Siegel, Fehst, Garger, \& Pick, 1980; Timmons \& Boudreau, 1978a, 1978b).

While the stereo tape systems can provide highfidelity/low-distortion DAF, their major disadvantage is that such systems are relatively expensive and lack portability. While there may be solid state devices commercially available (Hanson \& Metter, 1980), they are usually designed for specific needs and lack the variability of control necessary for general purposes.

This paper reports a relatively simple to construct portable solid state DAF device, requiring no audio tape, that allows for a continuously adjustable delay from 0 to $1,000 \mathrm{msec}$. The unit provides amplification and delay of a subject's voice by use of an integrated circuit (IC) device known as a Bucket Brigade Analog Delay Line. This device samples the analog input and transfers the samples "bucket-brigade" fashion to the output at a rate determined by the clock frequency.

\section{DESIGN CONSIDERATIONS}

The major objective in designing the solid state DAF was to build a device that could delay an auditory signal similar to the way tape recorders with a movable playback head could do, but without the problems to be expected with the use of audio tape when nonexpert individuals are using the equipment.

The equipment was designed to be used primarily by students and for demonstration purposes. Frequency response similar to that of a telephone, therefore, was considered adequate for those purposes.

The filter cutoff frequencies and characteristics were selected to provide acceptable voice quality while removing most or all of the clock pulses. At the longer delay settings (over $500 \mathrm{msec}$ ), however, the clock pulse is within audio range and is heard as a high-f requency tone. Providing a full $1 \mathrm{sec}$ of audio delay requires at least four delay-line sections to keep the clock frequency above voice range. Additional stages could be added to permit raising the clock pulses above the audio range, but a more complex oscillator would be required to provide the more desirable shorter delays. For most

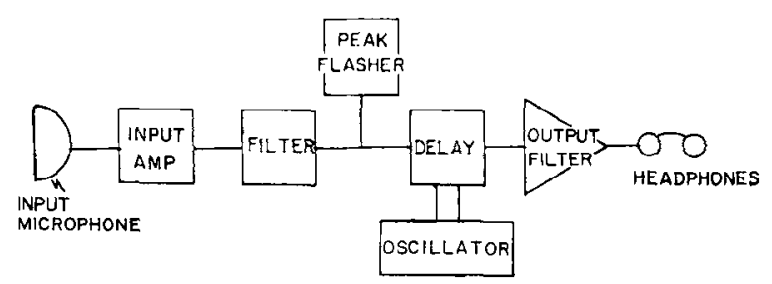

Figure 1. Block diagram for solid state delayed auditory feedback unit. 
general purposes, the high-frequency tone at longer delays does not interfere with the DAF effect.

\section{DESCRIPTION OF THE DAF UNIT}

The solid state DAF unit consists of three main sections in addition to the power supply: input amplifier/ filter, delay clock, and output filter amplifier. Figure 1 presents a block diagram for the major components of this unit.

\section{Input Amplifier/Filter}

The input section consists of two inverting IC audio amplifier stages providing approximately 40-dB gain. Input impedance is $10 \mathrm{kohm}$ and will accept any highimpedance microphone. Some high-pass filtering is provided by the $\mathrm{C} 1 / \mathrm{R} 1$ input network. This removes some of the "boominess" caused by the low-pass filters. Output of this amplifier drives a third-order noninverting low-pass filter. The cutoff frequency of $2,200 \mathrm{~Hz}$ was selected to remove frequencies above the voice

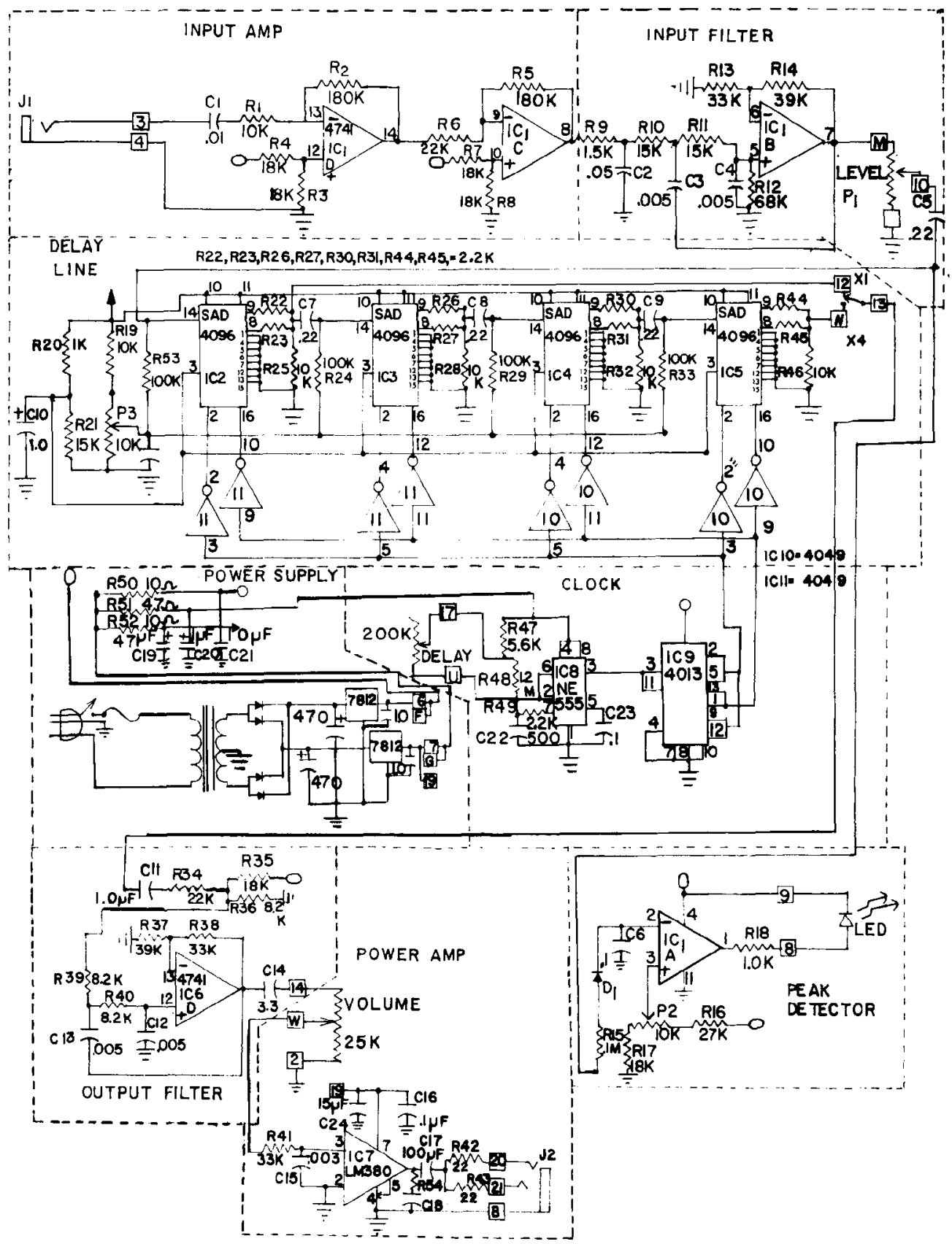

Figure 2. Delayed auditory feedback: Circuit diagram. 
range that would mix with the lower clock frequencies in the delay stages, causing unwanted beat notes.

A peak-detection circuit warns the operator if audio peaks approach the clipping level of the delay section. Since the voice is nonsymmetrical and 360-deg phase inverison takes place within the input stages, the peak detector monitors only positive peaks. This assumes the microphone is phased properly. Clipping in stages before the level control is not expected.

\section{Delay and Delay Clock}

The delay stage consists of four identical SAD-4096 delay line stages. A sample of audio is clocked into IC 2 on every Phase 1 clock pulse and transferred to the next stage on the next Phase 2 clock pulse. The sample appears 8192 clock pulses later at the output of IC 5 . A common control, P3, sets bias for lowest distortion. A front-panel switch labeled $\times 1 / x 4$ selects output from all four stages or from the first stage only to provide an expanded control range in the 10 - to 250 -msec range, in which the greatest DAF effect occurs.

The delay clock consists of an NE 555 pulse generator, with the pulse rate adjustable from $15 \mathrm{kHz}$ to $205 \mathrm{kHz}$. The output pulses are converted to squarewave clock signals by the 4013 dual flip-flop. The 4013 outputs are buffered by the two 4039 hex inverters, since clock-line capacitance of the SAD-4096 is quite high.

\section{Output Filter/Headphone Amplifier}

Either of two outputs from the delay line section is selected by the $\times 1 / \times 4$ switch. The $x 1$ selection allows for a range of audio delays from 0 to $250 \mathrm{msec}$, and the $\mathrm{x} 4$ selection allows for delays of from $250 \mathrm{msec}$ to $1,000 \mathrm{msec}$. Delayed audio combined with clock. frequency "noise" and beat tones is passed to a secondorder low-pass filter having a cutoff frequency of $3,900 \mathrm{~Hz}$. This filter smooths transitions between clock pulses remaining in the sampled audio and reduces objectionable beat notes from the output.

The final stage is a single-ended audio power amplifier IC that provides sufficient power gain to drive most commonly used headsets.

\section{Power Supply}

The power supply is a dual $12-\mathrm{V}$ regulated source. One section supplies power to the microphone amplifier stages. The other section supplies the remainder of the unit with each section decoupled through $\mathrm{R} / \mathrm{C}$ networks. A complete circuit diagram is presented in Figure 2.

\section{Construction}

The DAF unit is housed in a $7 \times 7 \times 3.5$ in. aluminum and plastic cabinet. It was built on a wire-wrap board with card-edge connections to the power supply and front-panel components. Some coupling of the clock pulses to low-level audio stages was experienced. Use of a well designed printed circuit would greatly reduce this coupling.

\section{ALIGNMENT PROCEDURE}

A copy of the alignment procedures for the complete solid state DAF unit may be obtained by writing Fred L. Yaffe, Department of Psychology, Washburn University, Topeka, Kansas 66621.

\section{DISCUSSION}

The solid state unit described in this paper provides a reliable, relatively inexpensive, flexible, and easy to operate method for studies and demonstrations of DAF. The compact basic unit is easy to construct and, with minor modifications of the power supply, can be made battery operated and totally portable. The estimated cost for all of the electrical components for the DAF unit is about $\$ 200$, with the major expense for the IC bucket-brigade devices. Additional expenses to be incurred are for a standard microphone for input and any common headphones for output.

\section{REFERENCES}

Allen, D. J., Antonitis, J. V., \& Magnzo, P. A. Reinforcing effects of prerecorded words and delayed speech feedback on the verbal behavior of a neologistic schizophrenic. Perceptual and Motor Skills, 1978, 46, 343-346.

Colling, J. K., \& Worthington, A. G. Delayed auditory feedback and cognitive complexity of tasks. American Journal of Psychology, 1978, 91, 93-99.

Doemaman, S., Landau, R., \& O'Conneld, D. The Stroop phenomenon: Perceptual conflict or response competition. Perceptual and Motor Skills, 1978, 47, 1127-1131.

ELIA8, J. The use of delayed auditory feedback in the identification of the left cerebral hemisphere as a temporal/duration processor. Journal of Auditory Research, 1977, 17, 155-160.

Hanson, W., \& METTEn, E. DAF as instrumental treatment for dysarthria in progressive supranuclear palsy: A case report. Journal of Speech and Hearing Disorders, 1980, 45, 268-276.

LECHNER, B. The effects of delayed auditory feedback and masking on the fundamental frequency of stutterers and nonstutterers. Journal of Speech and Hearing Research, 1979, 22, 343-353.

Lozano, R., \& Dreyer, D. Some effects of delayed auditory feedback on dyspraxia of speech. Journal of Communication Disorders, 1978, 11, 407-415.

Martin, R., \& Haroldson, S. Effects of five experimental treatments on stuttering. Journal of Speech and Hearing Research, 1979, 22, 132-146.

Sizget, G., Fehst, C., Garber, S., \& Pick, H. Delayed auditory feedback with children. Journal of Speech and Hearing Research, $1980,23,802-813$.

Stephens, S., \& Haggard, M. Acoustic properties of masking/ delayed feedback in the fluency of stutterers and controls. Journal of Speech and Hearing Research, 1980, 23, 527-538.

Timmons, B., \& Boudrenu, J. Delayed auditory feedback and the speech of stuttering and non-stuttering children. Perceptual and Motor Skills, 1978, 46, 551-555. (a)

Timmons, B., \& Boudreau, J. Speech disfluencies and delayed auditory feedback reactions of stuttering and non-stuttering children. Perceptual and Motor Skills, 1978, 47, 859-862. (b)

(Received for publication October 19, 1982; revision accepted December 30, 1982.) 